\title{
Tech transfer fails to translate into startups
}

Stacy Lawrence

In 2005 , US research institutions brought in $<\$ 2$ billion in license income. In its annual survey, the Association of University Technology Managers (AUTM) estimates tech transfer resulted in 527 new products

and 628 new firms in 2005, about one-sixth of which were life-science

\section{New biotech company formation}

Startup formation in 2005 remained sluggish across the world

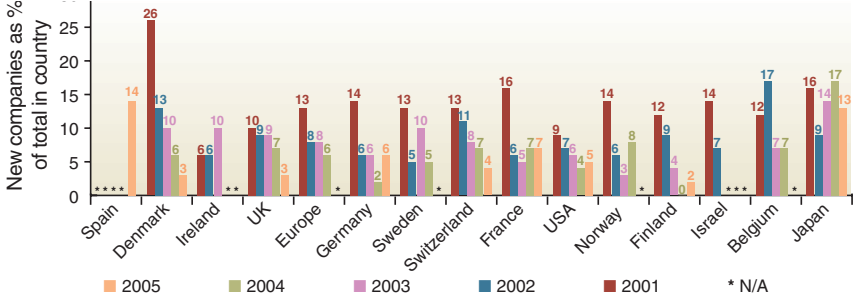

Source: Critical I, Japan Bioindustry Association, BIO, BioCOM/biotechnology.de

\section{US research institution R\&D spend and license income}

Average licensing income was up again in 2005, although R\&D spending increased, the trend of smaller increments continued.
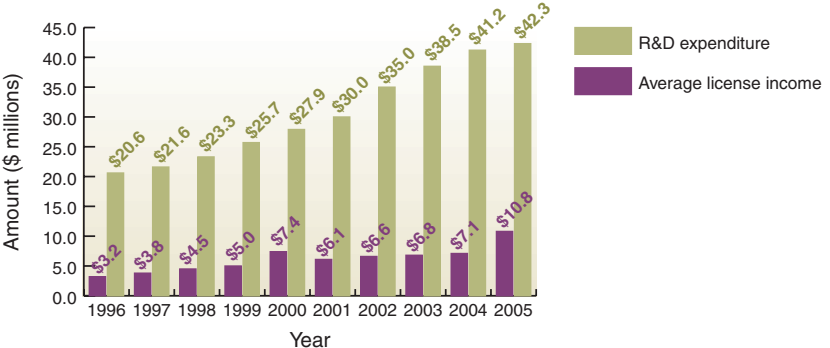

Research institutions include universities, hospitals and research institutes. Source: Association of University Technology Managers

Tech transfer at top US research institutions in 2005

\begin{tabular}{|c|c|c|c|c|c|c|c|}
\hline Universities & $\begin{array}{c}\text { R\&D spend } \\
(\$ \text { millions })^{\mathrm{a}}\end{array}$ & $\begin{array}{l}\text { License income } \\
(\$ \text { millions })^{\mathrm{b}}\end{array}$ & $\begin{array}{l}\text { Executed } \\
\text { licenses }\end{array}$ & $\begin{array}{c}\text { Cumulative } \\
\text { active licenses }\end{array}$ & Startups & $\begin{array}{l}\text { Patents } \\
\text { issued }^{\text {d }}\end{array}$ & $\begin{array}{c}\text { Patent } \\
\text { applications }\end{array}$ \\
\hline University of California System & 2,917 & 93 & 265 & 1,654 & 19 & 310 & 601 \\
\hline Johns Hopkins University & 1,674 & 12 & 94 & 803 & 5 & 81 & 318 \\
\hline Massachusetts Institute of Technology (MIT) & 1,133 & 40 & 93 & 794 & 20 & 133 & 290 \\
\hline University of Washington/Washington Research Foundation & 895 & 29 & 109 & 740 & 4 & 40 & 84 \\
\hline University of Illinois, Chicago, Urbana & 818 & 7 & 63 & 308 & 7 & 65 & 134 \\
\hline University of Wisconsin at Madison & 798 & 49 & 216 & 963 & 4 & 89 & 203 \\
\hline University of Michigan & 778 & 17 & 86 & 331 & 7 & 80 & 133 \\
\hline State University of New York Research Foundation & 736 & 14 & 84 & 372 & 6 & 35 & 128 \\
\hline University of Pennsylvania & 673 & 7 & 83 & 466 & 9 & 37 & 449 \\
\hline Penn State University & 638 & 2 & 21 & 163 & 3 & 37 & 90 \\
\hline \multicolumn{8}{|l|}{ Hospitals and research institutes } \\
\hline Massachusetts General Hospital & 483 & 95 & 140 & 554 & 5 & 56 & 165 \\
\hline Mayo Foundation for Medical Education and Research & 399 & 16 & 75 & 730 & 3 & 30 & 88 \\
\hline Brigham \& Women's Hospital, Inc. & 371 & 7 & 32 & 173 & 3 & 30 & 47 \\
\hline M.D. Anderson Cancer Center & 342 & 4 & 19 & 96 & 0 & 22 & 42 \\
\hline Sloan Kettering Institution for Cancer Research & 253 & 67 & 31 & 204 & 2 & 21 & 28 \\
\hline Fred Hutchinson Cancer Research Center & 226 & 2 & 31 & 120 & 2 & 3 & 15 \\
\hline Beth Israel Deaconess Medical Center & 191 & 2 & 18 & 120 & 4 & 10 & 30 \\
\hline Dana-Farber Cancer Institute & 187 & 6 & 21 & 323 & 1 & 13 & 36 \\
\hline Cleveland Clinic Foundation & 184 & 10 & 33 & 125 & 4 & 4 & 116 \\
\hline Children's Hospital, Cincinnati & 174 & 2 & 20 & 44 & 0 & 8 & 62 \\
\hline
\end{tabular}

174
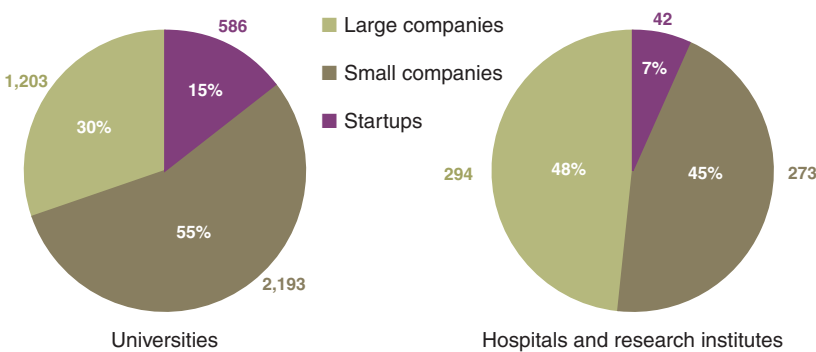

Hospitals and research institutes

Source: Association of University Technology Managers

based. Although AUTM estimates life sciences were responsible for about one-sixth of all licenses or license income, they accounted for only $8 \%$ of US startups. Other data indicate startup activity was poor across the globe.

\section{NIH technology transfer activities}

Invention disclosures and new patent applications for $\mathrm{NIH}$-funded research are in decline.

Invention disclosures

New patent applications filed

Total US patent applications

Source: National Institutes of Health

\section{Type of company obtaining licenses}

2005, around half of all US licenses went to small (biotech) companies; universities grant licenses to more startups compared with other institutions. 